\title{
Fear of falling and physical activity in hemodialysis patients: a pilot study
}

\author{
Nobuyuki Shirai ${ }^{1,2}$, Suguru Yamamoto ${ }^{2 *}$, Yutaka Osawa $^{3}$, Atsuhiro Tsubaki ${ }^{4}$, Shinichiro Morishita ${ }^{5}$, \\ Sumiyo Nitami ${ }^{6}$ and Ichiei Narita ${ }^{2}$
}

\begin{abstract}
Background: Hemodialysis (HD) patients are at a high risk of falls and fractures. The amount of physical activity (PA) they perform may be limited by psychosomatic factors associated with fear of falling, leading to frailty progression. This study aimed to clarify the relationship between fear of falling and PA in patients undergoing HD.

Methods: This cross-sectional study included $46 \mathrm{HD}$ patients. Fear of falling was evaluated using the Modified Falls Efficacy Scale (MFES). A 3-axis accelerometer was used to measure PA, including number of steps; 1 to 1.9 METs = static PA, 2 to $2.9=$ light PA, and 3 or more = moderate to vigorous PA (MVPA). We examined correlation of MFES with each type of PA. Factors affecting fear of falling were determined using multiple regression analysis.

Results: The median MFES was 9.2 (7.4, 10.0). MFES was associated with the number of steps $(r=0.608, p<0.001)$, light PA $(r=0.421, p=0.004)$, and MVPA $(r=0.546, p<0.001)$. Eighteen participants (39.1\%) experienced at least one fall in a year and had lower MFES than the non-fall group (fall group: 7.4 [5.1, 9.0] vs. non-fall group: 9.7 [8.5, 10.0], $p<0.001)$. Multiple regression analysis showed that MFES was independently associated with the number of steps $(B=279.7,95 \%$ confidence interval $[C l]=90.5-469.0, p=0.005)$ and MVPA $(B=3.52,95 \% \mathrm{Cl}=1.14-5.90, p=0.005)$, respectively.
\end{abstract}

Conclusions: Fear of falling was associated with amount of PA among patients undergoing HD. Interventions that target the fear of falling may be effective in reducing HD patients' fall risk by enhancing PA.

Keywords: Hemodialysis, Fear of falling, Physical activity, Triaxial accelerometer, Fall

\section{Background}

Hemodialysis (HD) patients experience fractures more frequently than healthy individuals $[1,2]$. Moreover, HD patients frequently experience falls that induce fractures [3]. Among older adults, reduced physical activity (PA) is associated with both falls and fractures [4], while HD patients may have poor PA owing to sarcopenia/frailty and dialysis treatment.

\footnotetext{
*Correspondence: yamamots@med.niigata-u.ac.jp

${ }^{2}$ Division of Clinical Nephrology and Rheumatology, Niigata University Graduate School of Medical and Dental Sciences, 1-757 Asahimachi-dori, Niigata 951-8510, Japan

Full list of author information is available at the end of the article
}

Psychological factors may be associated with PA and falls [5]. Fear of falling is a psychological state as ongoing concern about falling that ultimately leads to avoidance of the performance of daily activities [6]. Severe fear of falling restricts activity of daily living and PA even if patients have enough physical functions. The modified falls efficacy scale (MFES) is one of psychological parameters used to evaluate associated fall risk in the general population [7] while there is little data for MFES in HD patients.

HD patients engage in less PA [8] and demonstrate more psychological problems [9] than the general population; however, there are no reports to examine fear of falling and the association with PA by intensity in HD patients. This study evaluated the degree of fear of falling original author(s) and the source, provide a link to the Creative Commons licence, and indicate if changes were made. The images or other third party material in this article are included in the article's Creative Commons licence, unless indicated otherwise in a credit line to the material. If material is not included in the article's Creative Commons licence and your intended use is not permitted by statutory regulation or exceeds the permitted use, you will need to obtain permission directly from the copyright holder. To view a copy of this licence, visit http://creativecommons.org/licenses/by/4.0/. The Creative Commons Public Domain Dedication waiver (http://creativeco mmons.org/publicdomain/zero/1.0/) applies to the data made available in this article, unless otherwise stated in a credit line to the data. 
with MFES and detailed PA with 3D accelerometer, and examined their relationship among HD patients.

\section{Methods}

\section{Study design and participants}

This was a cross-sectional study conducted in a single hospital. From January 2017 to March 2017, we recruited all patients undergoing HD at the Niigata Rinko Hospital. To be eligible, persons had to be HD patients, aged over 20 years, on HD treatment for more than 1 year, and who provided consent to participate in this study. This study excluded persons who could not walk unassisted, recorded less than 10 activity meter hours [10], had lower limb amputations, and were on HD for less than 1 year. Age, sex, height, dry weight (DW), body mass index (BMI), dialysis duration, primary cause of kidney disease, presence of comorbidities (including cerebrovascular disease, cardiovascular disease, diabetes, and diabetic retinopathy), Charlson comorbidity index (CCI), serum albumin level, blood pressure, Kt/v for urea, blood hemoglobin level, and serum intact parathyroid hormone $(\mathrm{PTH})$ levels were reported as basic characteristics. The presence or absence of falls over the past year was recorded according to a previous report [11].

Study protocol complied with the 1975 Declaration of Helsinki, revised in 2013. Approval was granted by the Ethics Committee of Niigata Rinko Hospital (approval number 2104-97), and all patients provided written informed consent.

\section{Fear of falling evaluation}

MFES was used to assess fear of falling. It consists of 14 items associated with fall-related activities of daily living (ADL) and instrumental activities of daily living (IADL) (Table 1). Each item is scored on a 10-point numeric rating scale, in which $0=$ not confident, $5=$ fairly confident, and $10=$ completely confident, that means the lower score suggests the intense fear of falling [12]. The overall score is calculated as the average of all questions.

\section{Physical activity evaluation}

The PA meter Active Style Pro HJA-750C with built-in 3D accelerometer (OMRON Co., Tokyo, Japan) was used to assess the amount of PA among HD patients. The evaluation items consisted of the number of steps. Overall, PA was categorized as follows: 1 to 1.9 METs included sitting to standing was static PA; 2 to 2.9 METs included slow walking, etc. was light PA; and 3 METs or more involving activities more intense than normal walking was moderate to vigorous PA (MVPA) [13]. The activity meter recorded their physical activities for 4 days including 2 dialysis days and 2 non-dialysis days.
Table 1 Items in the Modified Falls Efficacy Scale (MFES)

\begin{tabular}{l} 
1. Get dressed and undressed \\
\hline 2. Prepare a simple meal \\
3. Take a bath or a shower \\
4. Get in/out of a chair \\
5. Get in/out of bed \\
6. Answer the door or telephone \\
7. Walk around the inside of your house \\
8. Reach into cabinets or closets \\
9. Light housekeeping \\
10. Simple shopping \\
11. Using public transport \\
12. Crossing roads \\
13. Light gardening or hanging out the laundry \\
14. Using front or rear steps at home
\end{tabular}

\section{Statistical analysis}

Each measured value is presented as a median (interquartile range) and percentage (\%). The participants were divided into two groups by MFES consisting of the High MFES group and the Low MFES group, and compared background data with Mann-Whitney U test and Chisquare test. Spearman's rank correlation coefficient test was employed to investigate the relationship between MFES and PA. The Mann-Whitney $U$ test was used to compare the MFES in the fall and non-fall groups over the past year. A multiple regression analysis was performed to find the association between MFES and each PA parameter. The analysis was adjusted for age, sex, $\mathrm{CCI}$, and history of fall.

As a sub-analysis, each PA parameter on HD days was compared that on non-HD days with the Wilcoxon signed-rank test. MFES and each PA parameter in patients with diabetes were compared with those without diabetes with Mann-Whitney U test. IBM SPSS statistics version 27 was used, and values of $p<0.05$, were considered statistically significant.

\section{Results}

This study initially included $72 \mathrm{HD}$ outpatients who visited the Niigata Rinko Hospital. Of these, 46 met the eligibility criteria and we were able to measure their PA for 4 days. Table 2 presents the clinical characteristics of the participants. Participants' median age was 70.5 (65.0, 75.0) years, $18(39.1 \%)$ were males, and the median dialysis period was $7.5(3.0,10.0)$ years. Median MFES was $9.2(7.4,10.0)$ at baseline, and Fig. 1 shows the distribution. Fourteen participants (30.4\%) had an MFES of 10, suggesting more confidence in fall-related ADL/IADL and less fall likelihood, while 32 (69.6\%) 
Table 2 Participants' clinical characteristics

\begin{tabular}{|c|c|c|c|c|}
\hline & All $(n=46)$ & High MFES group ( $n=23$ ) & Low MFES group ( $n=23)$ & $p$ value \\
\hline Age (years) & $70.5(65.0,75.0)$ & $67.0(63.0,73.0)$ & $72.0(65.0,76.0)$ & 0.301 \\
\hline Men, $n(\%)$ & $18(39.1)$ & $10(43.5)$ & $8(34.8)$ & 0.763 \\
\hline $\mathrm{BMI}\left(\mathrm{kg} / \mathrm{m}^{2}\right)$ & $22.1(19.0,24.3)$ & $22.7(19.5,24.1)$ & $20.8(18.6,24.8)$ & 0.668 \\
\hline Dialysis duration (years) & $7.5(3.0,10.0)$ & $6.0(2.0,9.0)$ & $8.0(5.0,15.0)$ & 0.054 \\
\hline \multicolumn{5}{|l|}{ Comorbid conditions } \\
\hline Cerebrovascular disease, $n(\%)$ & $13(28.2)$ & $4(17.4)$ & $9(39.1)$ & 0.189 \\
\hline Cardiac disease, $n(\%)$ & $41(89.1)$ & $20(87.0)$ & $21(91.3)$ & 1.0 \\
\hline Diabetes mellitus, $n(\%)$ & $26(56.5)$ & $10(43.5)$ & $16(70.0)$ & 0.136 \\
\hline Diabetic retinopathy, n (\%) & $12(26.1)$ & $4(17.4)$ & $8(34.8)$ & 0.314 \\
\hline CCl (score) & $6.0(5.0,7.0)$ & $6.0(4.0,7.0)$ & $6.0(5.0,7.0)$ & 0.720 \\
\hline \multicolumn{5}{|l|}{ Primary kidney disease } \\
\hline Diabetic nephropathy, n (\%) & $13(29.5)$ & $10(43.5)$ & $3(13.0)$ & 0.047 \\
\hline Glomerulonephritis, n (\%) & $18(36.4)$ & $9(39.1)$ & $9(39.1)$ & 1.0 \\
\hline Hypertension, $n(\%)$ & $11(25.0)$ & $9(39.1)$ & $2(8.7)$ & 0.035 \\
\hline Other nephropathies, n (\%) & $4(9.0)$ & $2(8.7)$ & $2(8.7)$ & 1.0 \\
\hline \multicolumn{5}{|l|}{ Laboratory values } \\
\hline Albumin (g/dL) & $3.5(3.2,3.6)$ & $3.5(3.2,3.7)$ & $3.4(3.3,3.6)$ & 0.642 \\
\hline Hemoglobin (g/dL) & $11.2(10.8,12.5)$ & $11.3(10.9,13.0)$ & $11.2(10.3,12.0)$ & 0.108 \\
\hline PTH $(p g / m L)$ & $172.0(172.0,286.8)$ & $169.0(133.0,338.0)$ & $175.0(78.0,264.0)$ & 0.423 \\
\hline Systolic blood pressure (mmHg) & $171.0(152.0,188.5)$ & $178.0(152.0,194.0)$ & $162.0(152.0,186.0)$ & 0.214 \\
\hline Diastolic blood pressure $(\mathrm{mmHg})$ & $87.0(77.0,99.8)$ & $92.0(80.0,101.0)$ & $81.0(74.0,98.0)$ & 0.129 \\
\hline $\mathrm{Kt} / \mathrm{V}$ & $1.5(1.3,1.8)$ & $1.5(1.2,1.6)$ & $1.6(1.3,1.8)$ & 0.191 \\
\hline History of falls, $n(\%)$ & $18(39.1)$ & $4(17.4)$ & $14(60.9)$ & 0.006 \\
\hline MFES (score) & $9.2(7.4,10.0)$ & $10.0(9.6,10.0)$ & $7.3(4.7,8.0)$ & $<0.001$ \\
\hline \multicolumn{5}{|l|}{ Physical activity } \\
\hline Steps (number/day) & $2081.3(662.3,2717.4)$ & $2723.5(2194.5,3850.8)$ & $921.5(492.3,1748.5)$ & $<0.001$ \\
\hline Static PA (min/day) & $605.3(508.6,724.3$ & $565.3(486.5,665.3)$ & $657.3(545.0,790.0)$ & 0.111 \\
\hline Light PA (min/day) & $98.3(85.6,135.6$ & $124.3(89.3,157.5)$ & $89.0(71.8,107.0)$ & 0.011 \\
\hline MVPA (min/day) & $31.3(18.7,48.8$ & $46.3(29.3,53.5)$ & $21.0(12.3,33.0)$ & $<0.001$ \\
\hline
\end{tabular}

BMI, body mass index; MFES, modified falls efficacy scale; MVPA, moderate to vigorous physical activity; PA, physical activity; PTH, parathyroid hormone

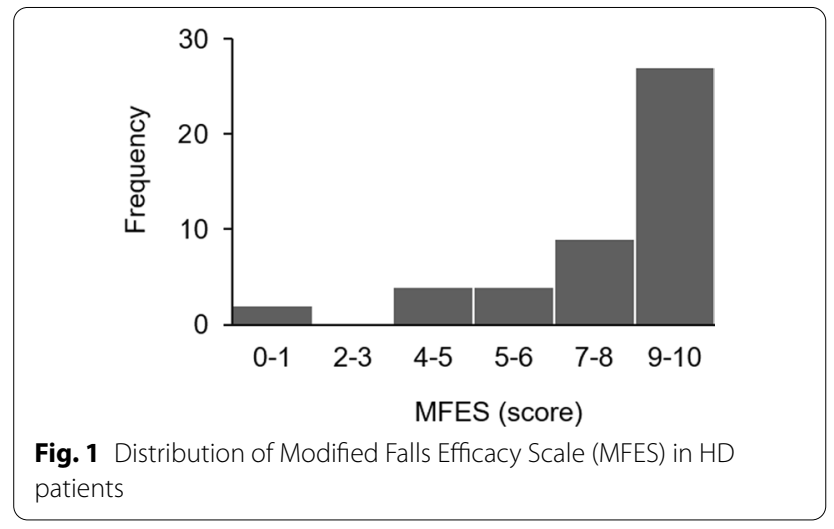

had an MFES less than 10, suggesting less confidence and more fall likelihood. There were two patients with lower MFES (0-1 point), and they had history of fall, cardiovascular disease, and diabetes. Figure 2 shows PA distribution among HD patients. There were 2081.3 (662.3, 2717.4) median steps per day, while the median minutes per day for static PA, light PA, and MVPA were 605.3 (508.6, 724.3), 98.3 (85.6, 135.6), and 31.3 (18.7, 48.8), respectively. The Low MFES group was shorter in height, had more diabetic nephropathy, had a greater history of falls, and had significantly fewer steps, light PA, and MVPA compared with the High MFES group. Patients with diabetes had lower MFES compared with those without diabetes, while there was no difference in each PA parameters among the groups (Additional file 1: Table S1). Figure 3 shows the relationship between MFES, PA, and history of falls. Positive correlations were observed between MFES and number of steps $(r=0.608, p<0.001)$, light PA $(r=0.421$, $p=0.004)$, and MVPA $(r=0.546, p<0.001)$. Eighteen 
A

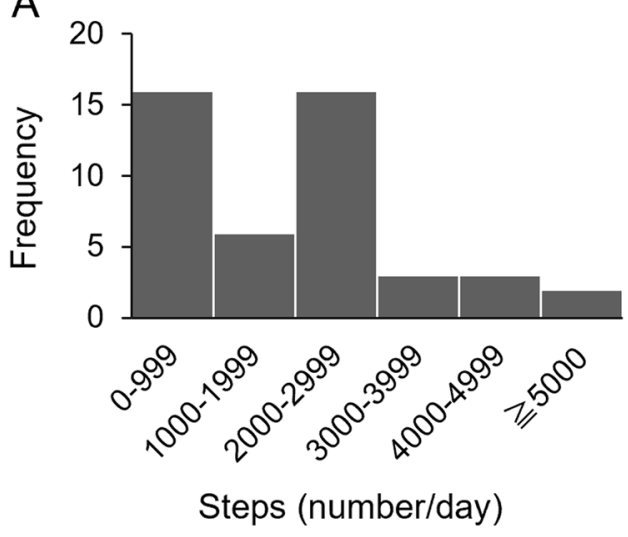

C

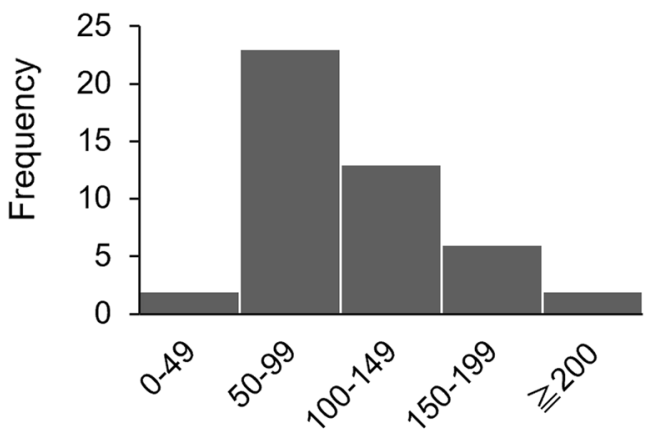

Light PA ( $\mathrm{min} /$ day)
B

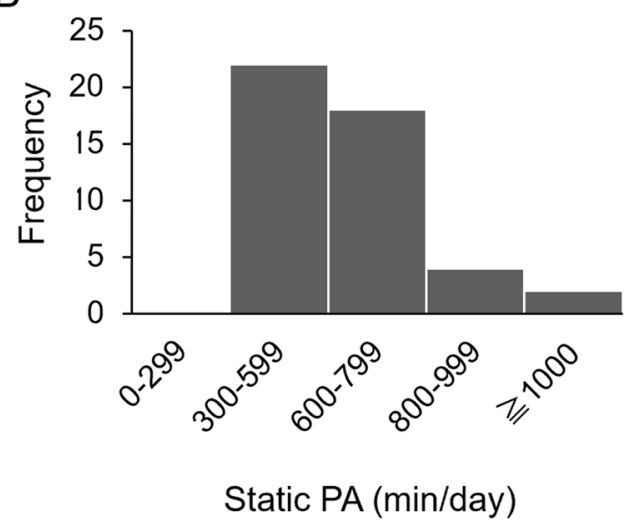

D

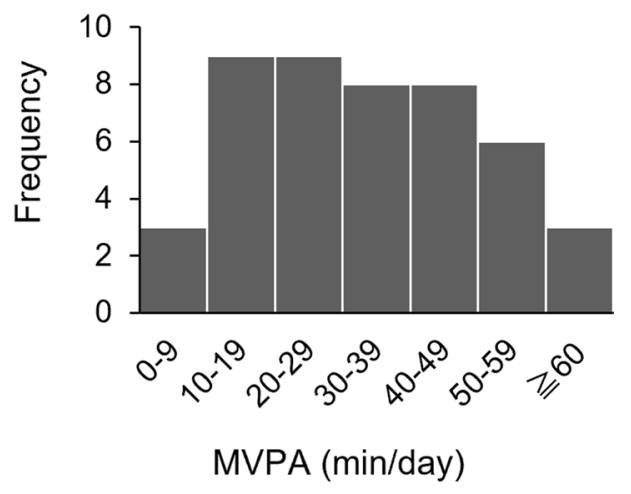

Fig. 2 Distribution of physical activity (PA) in HD patients. A Number of steps, $\mathbf{B}$ amount of static PA, C amount of light PA, and D amount of MVPA

participants $(39.1 \%)$ experienced at least one fall in that year and had lower MFES than the non-fall (fall group: $7.4[5.1,9.0]$ points vs. non-fall group: 9.7 [8.5, $10.0]$ points, $p<0.001)$. Results of univariate and multivariate analyses are summarized in Table 3 . Even after adjustment for age, sex, CCI and history of falls, MFES was consistently and significantly associated with steps $(B=279.7$, 95\% confidence interval $[\mathrm{CI}]=90.5-469.0$, $p=0.005)$ and MVPA $(B=3.52,95 \% \mathrm{CI}=1.14-5.90$, $p=0.005)$, respectively.

When we analyzed these categories according to HD days versus non-HD days, PA parameters were lower in the former than the latter (Additional file 1: Figure S1). Positive relations of the MFES were observed with the number of steps, light PA, and MVPA in non-HD days (Additional file 1: Figure S2) and the number of steps and MVPA in HD days (Additional file 1: Figure S3).

\section{Discussion}

This study examined the relationship between fear of falling, PA, and fall incidence in HD patients. The main findings were that MFES positively correlated with number of steps, light PA and MVPA. Previous studies involving the general older adult population have shown that fear of falling is associated with PA limitations [5, 14]. There is, however, no evidence about the prevalence of fear of falling and its association with PA and fall events among HD patients. The median MFES among HD patients in this study was $9.2(7.4,10.0)$, which was lower than that of healthy older adults of the same age, in an earlier report (9.76) [12]. This study used a 3-axis accelerometer to examine three levels of PA intensity. In addition to the association of MFES with number of steps, the total amount of PA, the association between MFES and PA was enhanced with PA intensity. (Table 3). These results suggest that HD patients had a severe fear of falling, which was associated with total PA, especially severe activity.

The deterioration of physical functions such as muscle strength and lower limb performance exacerbates fear of falling in the general population [15]. Moreover, it has been reported that PA of 3 METs or more is associated with physical function among healthy men and women, while 2 to 2.9 METs has been 
A

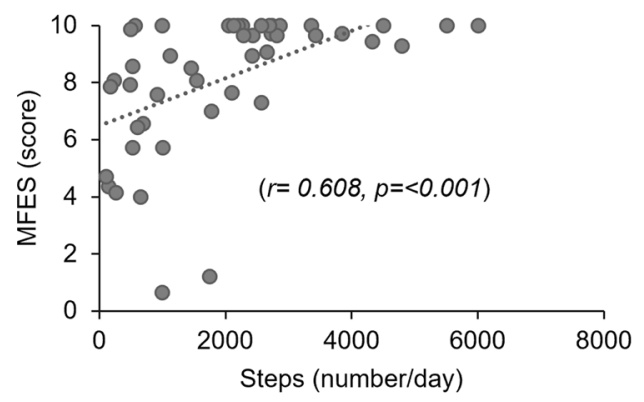

C

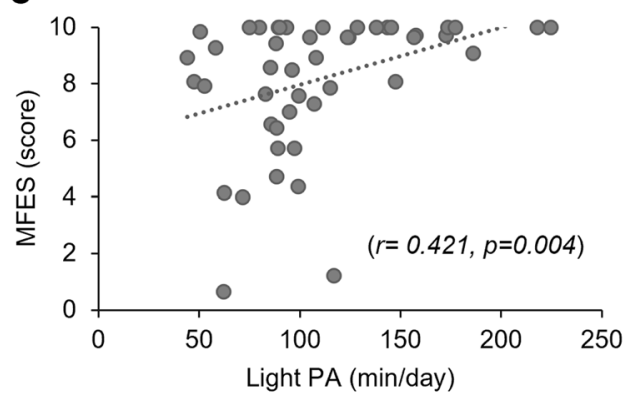

E

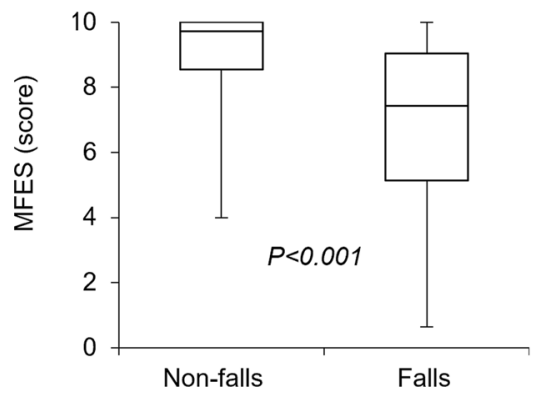

B

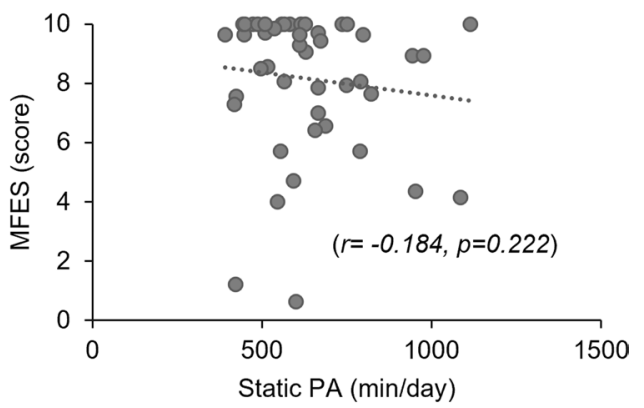

D

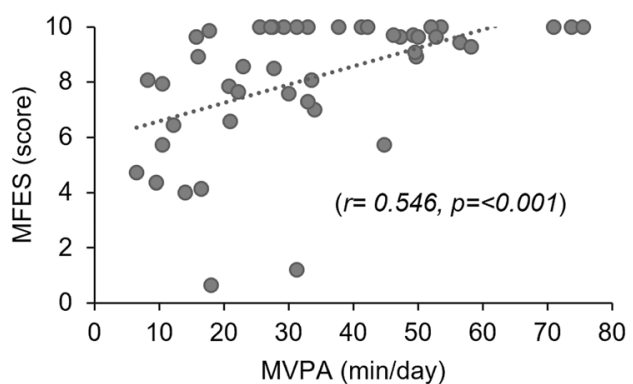

Fig. 3 Relationship between Modified Falls Efficacy Scale (MFES) scores and physical activity (PA) in HD patients. A Number of steps, B amount of static PA, C amount of light PA, and $\mathbf{D}$ amount of MVPA were shown via MFES. E Eighteen participants (39.1\%) experienced at least one fall in that year and had lower MFES than the non-fall (fall group: 7.4 [5.1, 9.0] points vs. non-fall group: $9.7[8.5,10.0]$ points, $p<0.001$ )

Table 3 Relationship between physical activity and fear of falling

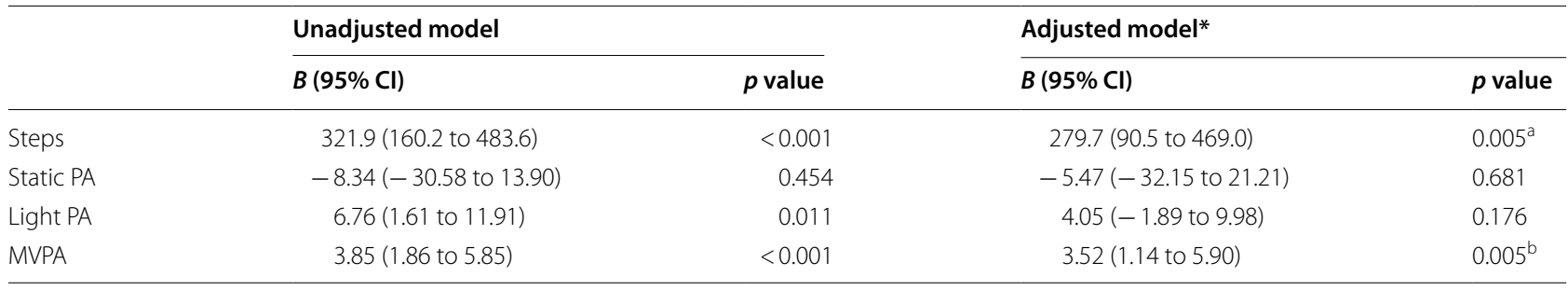

$\mathrm{Cl}$ : confidence interval, MVPA: moderate to vigorous physical activity, PA: physical activity

* Objective variable for Modified Falls Efficacy Scale. Adjusted for age, sex, Charlson comorbidity index, and history of falls

${ }^{\text {a Adjusted R2 }}=0.230$

${ }^{\mathrm{b}}$ Adjusted R2 $=0.187$ 
associated with physical function among women [10]. Research also suggests that patients with type 2 diabetes who engage in PA for $>150$ min per week have higher isometric knee extension muscle strength than those with $<150 \mathrm{~min}$ of activity [16]. Among dialysis patients, accelerometer-measured PA was correlated with a 6-min walking time [8]. It is, therefore, possible that, among HD patients, fear of falling is associated with fall risk owing to the impairment of physical function. Psychosomatic factors may also be a major contributor to the fear of falling. It is well known that psychosomatic problems, including depression and anxiety, are frequently observed among HD patients [9]. Moreover, antidepressants use is associated with increased fractures in that demographic [17]. Thus, it is possible that fear of falling caused by psychosomatic problems is associated with a higher fall risk, which may lead to increased fracture incidence among HD patients.

All kinds of PA were found to be greater on non-HD days than HD days (Additional file 1: Figure S1). The PA decrease on HD days is thought to be the result of weakness and fatigue post-HD treatment and the loss of potential activity time owing to HD sessions [18]. Concerning the former, several dialysis-related factors, such as changes in blood pressure [19] and fluid volume [20], may limit PA on HD days.

Exercise interventions reportedly reduce fear of falling among older adults in the community [21]. This study found the mean number of daily steps taken to be 2081.3. This suggests that additional exercise is needed to improve fear of falling and PA, as the recommended number of steps on non-HD days is 4000 or more [22]. Further studies are needed to assess the exercise intervention's potential impact on fear of falling toward the reduction of fall risk among HD patients.

It is, of course, necessary to consider limitations of this study. Since this was a small group cross-sectional study, it was impossible to clarify the causal relationship between fear of falling and PA. We did not examine the effect of CKD-related factors, such as nutrition and muscle strength, on the association between fear of falling and PA owing to small sample size, and future studies will be needed to be clarified it. It is also possible that some aspects of fall history were overlooked, as researchers verbally inquired about falls which took place in the past year. Although this was a pilot study with a small sample size, it is the first to demonstrate the severity of fear of falling while presenting a detailed account of PA in HD patients. In the future, large-scale prospective studies are needed to provide further insight into fear of falling and its association with PA.

\section{Conclusions}

Fear of falling was associated with number of steps and MVPA in HD patients. Psychological approach for fear of falling may effectively reduce fall risk in HD patients through PA enhancement.

\section{Abbreviations}

HD: Hemodialysis; PA: Physical activity; MFES: Modified Falls Efficacy Scale; MVPA: Moderate to vigorous PA; DW: Dry weight; BMl: Body mass index; PTH: Parathyroid hormone; ADL: Activities of daily living; IADL: Instrumental activities of daily living.

\section{Supplementary Information}

The online version contains supplementary material available at https://doi. org/10.1186/s41100-021-00383-3.

Additional file 1. Figure S1. Comparisons of physical activity (PA) on hemodialysis (HD) days and non-HD days, according to $(A)$ number of steps, (B) amount of static PA, (C) amount of light PA, and (D) amount of MVPA. Figure S2. Relationship between Modified Falls Efficacy Scale (MFES) scores, physical activity (PA) on non-hemodialysis (HD) days in HD patients. (A) number of steps, (B) amount of static PA, (C) amount of light PA, and (D) amount of MVPA. Figure S3. Relationship between Modified Falls Efficacy Scale (MFES) scores, physical activity (PA) on hemodialysis (HD) days in HD patients. (A) number of steps, (B) amount of static PA, (C) amount of light PA, and (D) amount of MVPA.

\section{Acknowledgements}

The authors acknowledge the expert comments and suggestions of Kou Kitabayashi.

\section{Authors' contributions}

N.S., S.Y., and I.N contributed to conception, study design, and data interpretation. N.S., S.Y., Y.O., A.T., M.S., and N.S. contributed to measurement and analyses. The manuscript was drafted by N.S., S.Y., and I.N. The final version of the manuscript was approved by N.S., S.Y., Y.O., T.A., M.S., N.S., and I.N. All authors read and approved the final manuscript.

Funding

The authors received no specific funding for this work.

\section{Declarations}

Ethics approval and consent to participate

Study protocol complied with the 1975 Declaration of Helsinki, revised in 2013. Approval was granted by the Ethics Committee of Niigata Rinko Hospital (Approval No. 2104-97), and all patients provided written informed consent.

\section{Consent for publication}

Not applicable.

\section{Competing interests}

The authors have no conflicts of interest to declare.

\section{Author details}

${ }^{1}$ Department of Rehabilitation, Niigata Rinko Hospital, Niigata, Japan. ${ }^{2}$ Division of Clinical Nephrology and Rheumatology, Niigata University Graduate School of Medical and Dental Sciences, 1-757 Asahimachi-dori, Niigata 951-8510, Japan. ${ }^{3}$ Internal Medicine, Niigata Rinko Hospital, Niigata, Japan. ${ }^{4}$ Institute for Human Movement and Medical Sciences, Niigata University of Health and Welfare, Niigata, Japan. ${ }^{5}$ Department of Physical Therapy, School of Health Science, Fukushima Medical University, Fukushima, Japan. ${ }^{6}$ Department of Rehabilitation, Niigata Bandai Hospital, Niigata, Japan. 
Received: 30 June 2021 Accepted: 6 November 2021

Published online: 18 November 2021

\section{References}

1. Tentori F, McCullough K, Kilpatrick RD, Bradbury BD, Robinson BM, Kerr $P G$, et al. High rates of death and hospitalization follow bone fracture among hemodialysis patients. Kidney Int. 2014;85(1):166-73. https:// doi.org/10.1038/ki.2013.279.

2. Wakasugi M, Kazama JJ, Wada A, Hamano T, Masakane I, Narita I. Longterm excess mortality after hip fracture in hemodialysis patients: a nationwide cohort study in Japan. J Bone Miner Metab. 2020;38(5):718-29. https://doi.org/10.1007/s00774-020-01110-4

3. López-Soto PJ, De Giorgi A, Senno E, Tiseo R, Ferraresi A, Canella C, et al. Renal disease and accidental falls: a review of published evidence. BMC Nephrol. 2015;16:176. https://doi.org/10.1186/s12882-015-0173-7.

4. Cauley JA, Harrison SL, Cawthon PM, Ensrud KE, Danielson ME, et al. Objective measures of physical activity, fractures and falls: the osteoporotic fractures in men study. J Am Geriatr Soc. 2013;61 (7):1080-8. https:// doi.org/10.1111/jgs.12326.

5. Jefferis BJ, lliffe S, Kendrick D, Kerse $N$, Trost $\mathrm{S}$, Lennon LT, et al. How are falls and fear of falling associated with objectively measured physical activity in a cohort of community-dwelling older men? BMC Geriatr. 2014;14:114. https://doi.org/10.1186/1471-2318-14-114.

6. Tinetti ME, Powell L. Fear of falling and low self-efficacy: a case of dependence in elderly persons. J Gerontic. 1993;48:35-8. https://doi.org/ 10.1093/geronj/48.Special_Issue.35.

7. Jung D. Fear of falling in older adults: comprehensive review. Asian Nurs Res. 2008;2(4):214-22. https://doi.org/10.1016/S1976-1317(09)60003-7.

8. Kim JC, Shapiro BB, Zhang M, Li Y, Porszasz J, Bross R, et al. Daily physical activity and physical function in adult maintenance hemodialysis patients. J Cachexia Sarcopenia Muscle. 2014;5(3):209-20. https://doi.org/ 10.1007/s13539-014-0131-4

9. Oyekçin DG, Gülpek D, Sahin EM, Mete L. Depression, anxiety, body image, sexual functioning, and dyadic adjustment associated with dialysis type in chronic renal failure. Int J Psychiatry Med. 2012;43(3):227-41. https://doi.org/10.2190/PM.43.3.c.

10. Izawa KP, Shibata A, Ishii K, Miyawaki R, Oka K. Associations of lowintensity light physical activity with physical performance in communitydwelling elderly Japanese: a cross-sectional study. PLOS ONE. 2017;12(6): e0178654. https://doi.org/10.1371/journal.pone.0178654.

11. Cook WL, Jassal SV. Prevalence of falls among seniors maintained on hemodialysis. Int Urol Nephrol. 2005;37(3):649-52. https://doi.org/10. 1007/s11255-005-0396-9.

12. Hill KD, Schwarz JA, Kalogeropoulos AJ, Gibson SJ. Fear of falling revisited. Arch Phys Med Rehabil. 1996;77(10):1025-9. https://doi.org/10.1016/ s0003-9993(96)90063-5.
13. Ainsworth BE, Haskell WL, Herrmann SD, Meckes N, Bassett DR Jr, TudorLocke C, et al. 2011 Compendium of physical activities: a second update of codes and MET values. Med Sci Sports Exerc. 2011:43(8):1575-81. https://doi.org/10.1249/MSS.0b013e31821ece12.

14. Schoene D, Heller C, Aung YN, Sieber CC, Kemmler W, Freiberger E. A systematic review on the influence of fear of falling on quality of life in older people: is there a role for falls? Clin Interv Aging. 2019;14:701-19. https:// doi.org/10.2147/CIA.S197857.

15. Trombetti A, Reid KF, Hars M, Herrmann FR, Pasha E, Phillips EM, et al. Age-associated declines in muscle mass, strength, power, and physical performance: impact on fear of falling and quality of life. Osteoporos Int. 2016;27(2):463-71. https://doi.org/10.1007/s00198-015-3236-5.

16. Shirai N, Tsubaki A, Morishita S, Honma D, Isobe S, Ikarashi K, et al. The association between time spent in performing physical activity and physical function in outpatients with type 2 diabetes who may have diabetic neuropathy. Diabetes Metab Syndr. 2020;14(6):2111-6. https:// doi.org/10.1016/j.dsx.2020.10.020

17. Ishida JH, McCulloch CE, Steinman MA, Grimes BA, Johansen KL. Psychoactive medications and adverse outcomes among older adults receiving hemodialysis. J Am Geriatr Soc. 2019;67(3):449-54. https://doi.org/10. 1111/jgs.15740.

18. Majchrzak KM, Pupim LB, Chen K, Martin CJ, Gaffney S, Greene JH, et al. Physical activity patterns in chronic hemodialysis patients: comparison of dialysis and nondialysis days. J Ren Nutr. 2005;15(2):217-24. https://doi. org/10.1053/j.jrn.2004.08.002.

19. Chou JA, Kalantar-Zadeh K. Volume balance and intradialytic ultrafiltration rate in the hemodialysis patient. Curr Heart Fail Rep. 2017;14(5):4217. https://doi.org/10.1007/s11897-017-0356-6.

20. Harada T, Obokata M, Kurosawa K, Sorimachi H, Yoshida K, Ishida H, et al. Relationships of high cardiac output with ventricular morphology, myocardial energetics, and energy costs in hemodialysis patients with preserved ejection fraction. Int J Cardiovasc Imaging. 2019;35(3):469-79. https://doi.org/10.1007/s10554-018-1472-4.

21. Gawler SJ, Gage H, Masud T, Bowling A, Pearl M, Morris RW, et al. Exercise for reducing fear of falling in older people living in the community. Cochrane Database Syst Rev. 2014;2014(11):CD009848. https://doi.org/ 10.1002/14651858.CD009848.pub2.

22. Matsuzawa R, Roshanravan B, Shimoda T, Mamorita N, Yoneki K, Harada $M$, et al. Physical activity dose for hemodialysis patients: where to begin? Results from a prospective cohort study. J Ren Nutr. 2018;28(1):45-53. https://doi.org/10.1053/j.jrn.2017.07.004

\section{Publisher's Note}

Springer Nature remains neutral with regard to jurisdictional claims in published maps and institutional affiliations.
Ready to submit your research? Choose BMC and benefit from:

- fast, convenient online submission

- thorough peer review by experienced researchers in your field

- rapid publication on acceptance

- support for research data, including large and complex data types

- gold Open Access which fosters wider collaboration and increased citations

- maximum visibility for your research: over 100M website views per year

At BMC, research is always in progress.

Learn more biomedcentral.com/submissions 